\title{
DEEP UNDERGROUND NEUTRINO EXPERIMENT
}

\author{
Robert J. Wilson (for the DUNE collaboration) ${ }^{a}$ \\ Department of Physics, Colorado State University, Fort Collins, CO 80523, USA
}

\begin{abstract}
The Deep Underground Neutrino Experiment (DUNE) collaboration will perform an experiment centered on accelerator-based long-baseline neutrino studies along with nucleon decay and topics in neutrino astrophysics. It will consist of a modular 40-kt (fiducial) mass liquid argon TPC detector located deep underground at the Sanford Underground Research Facility in South Dakota and a high-resolution near detector at Fermilab in Illinois. This configuration provides a $1300-\mathrm{km}$ baseline in a megawatt-scale neutrino beam provided by the Fermilabhosted international Long-Baseline Neutrino Facility.
\end{abstract}

\section{Introduction}

Neutrinos are the most abundant known matter particles in the Universe, but they are among the least well-understood of the stable particles. The observation that neutrinos produced as one type (flavor) may later interact as a different type provides evidence for the phenomenon of neutrino flavor mixing and for small, but nonzero, neutrino masses. The framework characterizing these observations is similar to that describing corresponding phenomena in the quark sector, but with a very different pattern of mixing angle values. As in the quark case, this framework involves a phase parameter, $\delta_{\mathrm{CP}}$, that changes sign under combined charge conjugation and parity $(\mathrm{CP})$ reversal operations and thus would lead to CP symmetry-violating asymmetries between the pattern of oscillations for neutrinos and antineutrinos. While groundbreaking on its own, the observation of such asymmetries would also provide an experimental underpinning for the basic idea of leptogenesis as an explanation for the baryon asymmetry of the universe [1].

Neutrino oscillation data to date tell us about the magnitudes of the differences in the squared masses of the neutrino mass states, and about the sign of the mass-squared difference between the two states that are closest in mass, but not about the difference of that pair with respect to the third, which may be heavier (normal ordering) or lighter (inverted ordering) than the other two. Resolving this neutrino mass hierarchy ambiguity, along with precise measurements of neutrino mixing angles, would have significant theoretical, cosmological and experimental implications. Because the forward scattering of neutrinos in matter alters the oscillation pattern in a hierarchy-dependent way, GeV-scale neutrinos traveling several hundred kilometers through the Earth's crust enables a decisive determination of the hierarchy independent of the value of $\delta_{\mathrm{CP}}$. Taken together, such a comprehensive set of measurements will thoroughly test the standard three-neutrino flavor paradigm that guides our current

\footnotetext{
${ }^{a}$ E-mail: wilson@colostate.edu
} 


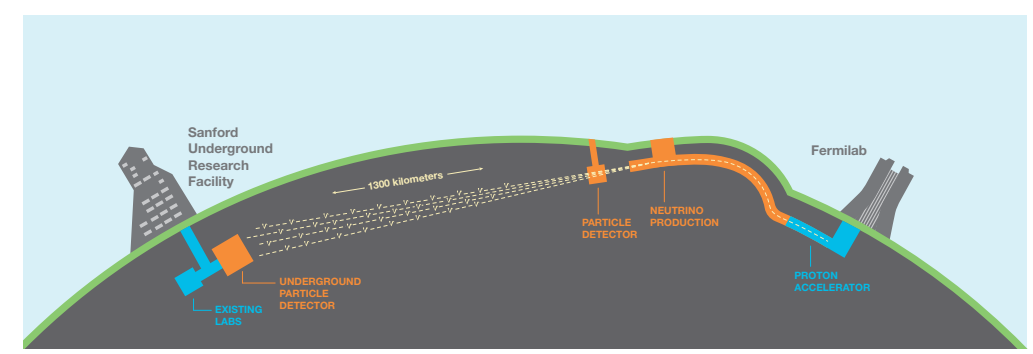

Figure 1: Long-Baseline Neutrino Facility/Deep Underground Neutrino Experiment.

understanding and will provide greatly extended sensitivity to signatures for nonstandard neutrino interactions in matter.

Massive detectors deep underground have broad discovery potential beyond the accelerator neutrino program. The observation of nucleon decay would be a watershed event for the understanding of physics at high energy scales. Neutrinos from supernovae would provide key insights into the physics of gravitational collapse and may also reveal fundamental properties of the neutrino.

The Deep Underground Neutrino Experiment (DUNE) science program and the Long-Baseline Neutrino Facility, as illustrated schematically in Figure 1, are very well described in the Conceptual Design Report (CDR) [2], here I present just a few highlights from that report.

\section{Experiment Method and Measurement Sensitivities}

The essential method of long-baseline experiments is to produce a (nearly) pure muon-neutrino beam with an energy spectrum matched to the oscillation pattern at the chosen distance (the baseline) then measure the spectrum of muon-neutrinos and electron-neutrinos at the distant detector. DUNE has chosen an optimal beam and distance to have sensitivity to $\mathrm{CP}$ violation, $\mathrm{CP}$ phase, neutrino mass hierarchy, and other oscillation parameters in the same experiment [3].

Figure 2 shows the disappearance and appearance spectra that would be observed on axis in a liquid argon detector at a distance of $1300 \mathrm{~km}$ from a neutrino (antineutrino) source with an exposure of 150 kt.MW.yr; for example, a 40-kt fiducial mass detector exposed to a $1.2 \mathrm{MW}, 80 \mathrm{GeV}$ proton beam for 3 years. Spectra for $\delta_{\mathrm{CP}}=0$ and normal mass hierarchy are shown for the CDR design along with a recent optimized beam design. The full power of the experiment comes from a simultaneous fit to these spectra.

\subsection{Mass Hierarchy, Charge-Parity Violation and the Measurement of $\delta_{\mathrm{CP}}$}

The $1300 \mathrm{~km}$ baseline establishes one of DUNE's key strengths: sensitivity to the matter effect. This effect leads to a large discrete asymmetry in the neutrino 

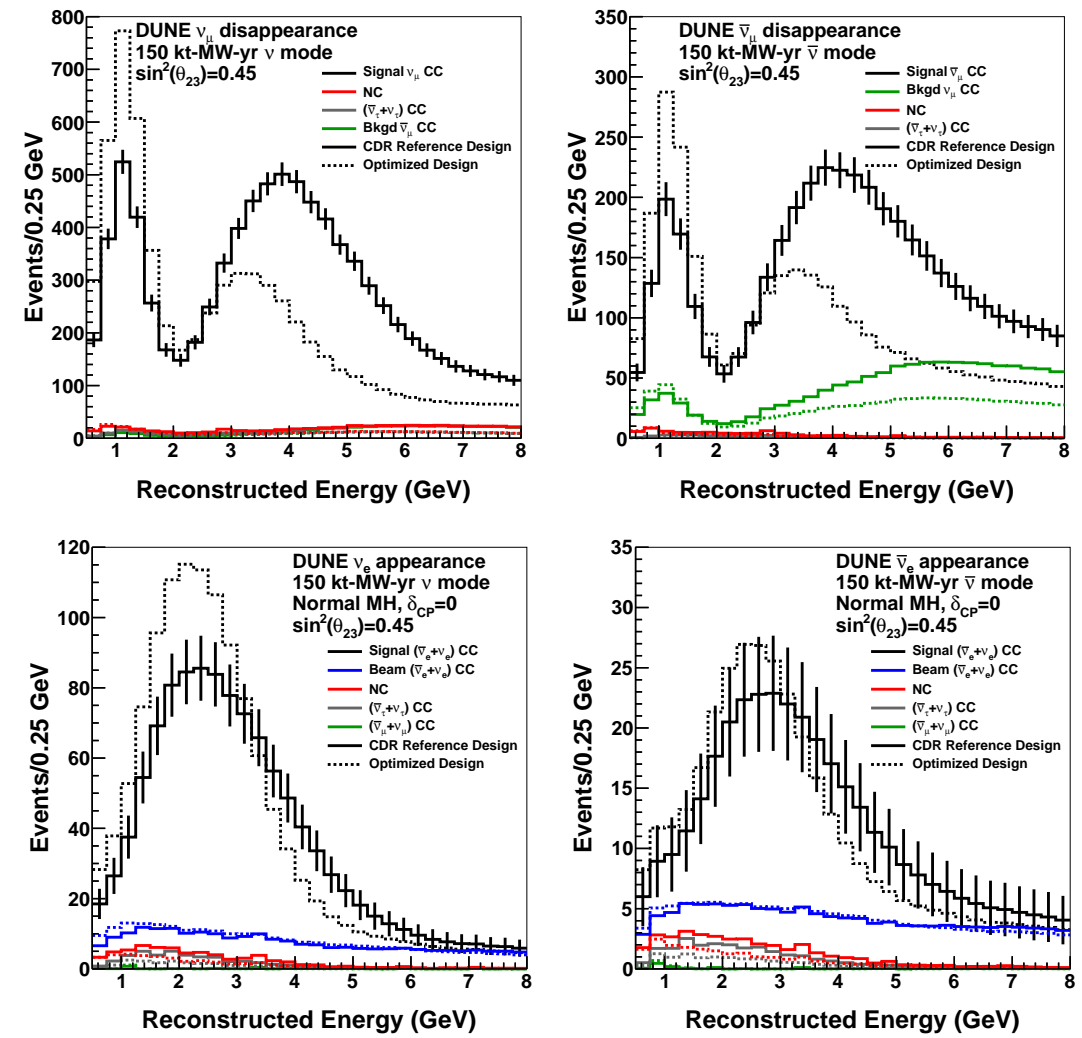

Figure 2: Disappearance (top) and appearance (bottom) spectra for neutrinos (left) and antineutrinos (right).

and antineutrino oscillation probabilities, the sign of which depends on the mass hierarchy $(\mathrm{MH})$. Figure 3[left] shows the mass hierarchy determination significance as a function of the unknown CP phase angle for a total exposure of 300 kt.MW.yr; unambiguous determination is achieved for any value of the phase angle. For some values this discovery arrives much sooner.

The DUNE program has two somewhat distinct objectives with regard to CP symmetry violation in the oscillation channel. First, DUNE aims to make a precise determination of the value of $\delta_{\mathrm{CP}}$ within the context of the standard three-flavor mixing scenario described by the PMNS matrix. Second, and perhaps more significantly, DUNE aims to observe a signal for leptonic CP violation, independent of the underlying nature of neutrino oscillation phenomenology. Together, the pursuit of these two goals provides a thorough test of the standard three-flavor scenario. 

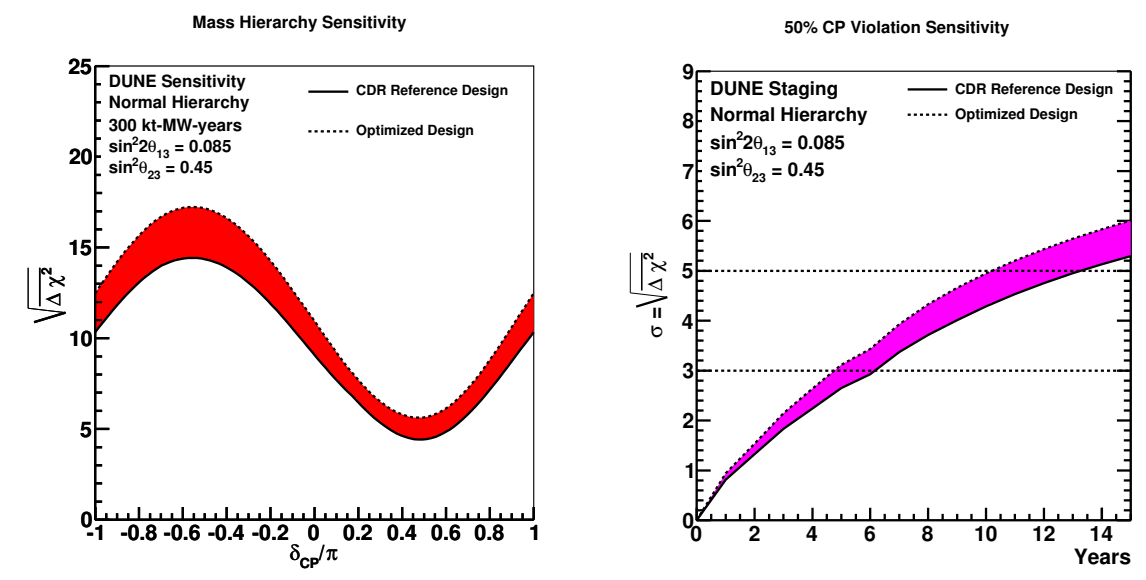

Figure 3: [Left] Mass hierarchy discrimination metric plotted as a function of $\delta_{\mathrm{CP}}$ for a $300 \mathrm{kt} \cdot \mathrm{MW} \cdot y e a r$ exposure. [Right] Significance with which CP violation can be determined for $50 \%$ of $\delta_{\mathrm{CP}}$ values as a function of exposure in years using a draft staging plan. The shaded region represents the range in sensitivity due to variations in the beam design.

Figure 3[right] shows the expected sensitivity to non-zero CP phase angle as a function of calendar time for a draft staging scenario outlined in the CDR physics section [2]. Five-sigma determination of $\mathrm{CP}$ violation in half the possible phase space is achieved in about 10 years with an optimized horn focusing system under development. The $1 \sigma$ resolution for $\delta_{\mathrm{CP}}$ will be $10^{\circ}-$ $18^{\circ}$ (depending on the value of $\delta_{\mathrm{CP}}$ ) after an exposure of $300 \mathrm{kt} . \mathrm{MW} . \mathrm{yr}$ and ultimately approaches a precision better than $10^{\circ}$, which is comparable to the current precision on the CP phase in the CKM matrix in the quark sector. Concurrent analysis of the corresponding atmospheric-neutrino samples will improve the precision with which the mass hierarchy is resolved, which, when combined with the beam data, will increase the $\mathrm{CP}$ violation sensitivity at roughly the $1 \sigma$ level after 10 years operation.

\subsection{Deep Underground Science}

Among massive detectors designed for neutrino and nucleon decay physics, the LArTPC technology offers unmatched capabilities for position and energy resolution and for high-precision reconstruction of complex interaction topologies over a broad energy range. These features in a detector deep underground will provide a powerful complementarity to the large, underground water Cherenkov or scintillator-based detectors that may be operating at the same time. For example, LArTPC detectors are especially well suited to supersymmetry-favored proton decay modes and uniquely provide detection efficiency and background rejection sufficient to enable a discovery with a single well-reconstructed event. 
There is also complementarity with respect to supernova-neutrino detection since liquid argon detectors are primarily sensitive to the electron-neutrino component of the flux, while anti-electron-neutrino interactions dominate for water and scintillator-based detectors. Thus, DUNE will be sensitive to different features of the supernova-neutrino production process. G. Raffelt addresses this topic in more detail in his presentation at this conference. Finally, the LArTPC technology located deep underground opens up an avenue for precision studies of oscillation physics with atmospheric neutrinos, thereby augmenting the results of the beam-based measurements at the core of the experiment.

\section{Long-Baseline Neutrino Facility and the DUNE Detectors}

The Long-Baseline Neutrino Facility (LBNF) is an international facility hosted in the US - it consists of the neutrino beamline and conventional facilities for both the near and far site detector complexes. The far site is planned for the Sanford Underground Research Facility located at the former Homestake Mine in Lead, South Dakota, well known for the Davis experiment that revolutionized our thinking about neutrinos. Figure 4 shows a schematic of the planned configuration for the DUNE far detector at the 4850 level (4850' below the surface); it consists of four cryostats with dimensions $15(\mathrm{w}) \times 14(\mathrm{~h}) \times 62(\mathrm{l}) \mathrm{m}^{3}$, each able to contain a total mass of about 17,000 tonnes of liquified argon.

A new neutrino beamline at Fermilab will provide initial beam power of 1.2 MW, enabled by upgrades to the front end of the accelerator complex carried out within the Proton Improvement Plan-II (PIP-II) program. In anticipation of potential enhancements beyond PIP-II, the beamline is designed to support upgrades to accommodate a beam power of $2.3 \mathrm{MW}$.

A highly capable near detector will measure the absolute flux and energy scales of all four neutrino species in the LBNF beam, as well as neutrino cross sections on argon, water, and other nuclear targets in the beam's energy range. These measurements are needed to control the systematic uncertainties to a level consistent with the ultimate desired precision for the oscillation parameter measurements. The near detector will also enable a broad range of precision neutrino-interaction measurements, thereby adding a compelling scientific program of its own.

The current baseline near detector design consists of a low-mass straw-tube tracker with radiator foils and pressurized gaseous argon target immersed in a magnetic field and is located about $460 \mathrm{~m}$ from the neutrino target. Additional target materials, such as calcium and carbon, can be embedded within the finegrained tracker. At nominal beam power this detector would collect roughly 10 million neutrino interactions per year, enabling high precision relative and absolute flux measurements. Other options, such as a LArTPC (possibly magnetized) and a high-pressure argon gas TPC are also under consideration. The 


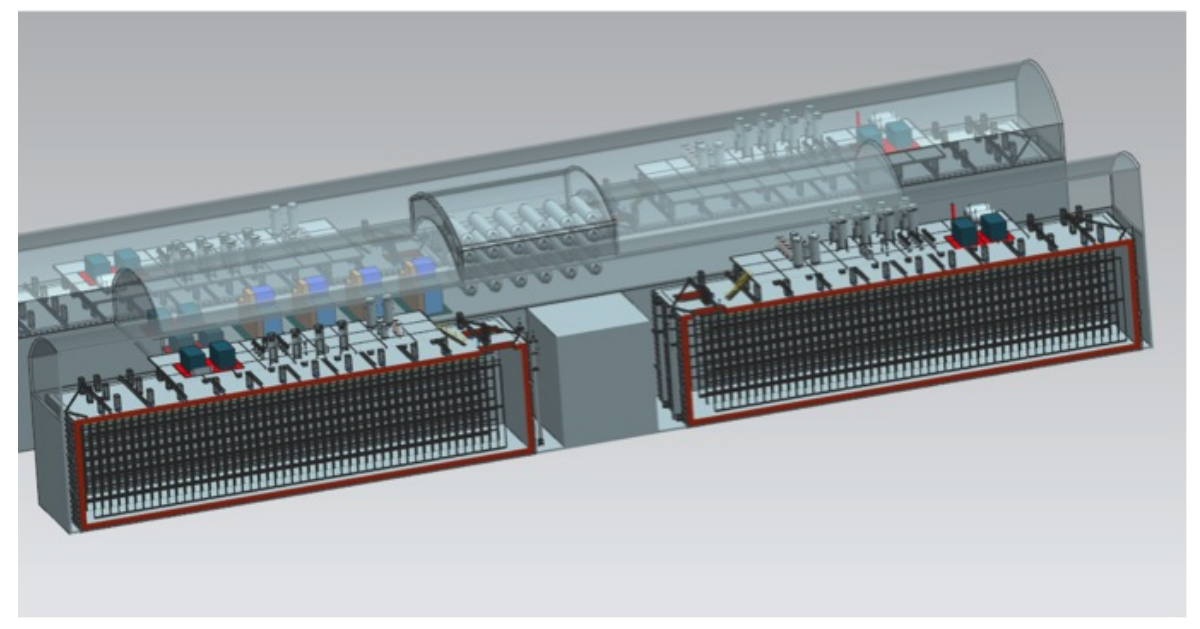

Figure 4: Far site 40-kt fiducial mass detector configuration consisting of four 10-kt liquid argon Time Projection Chambers.

near neutrino detector will be complemented by a system of tertiary beam muon detectors located downstream of the LBNF beamline absorber.

The 10-kt DUNE reference design TPC [4] builds on the pioneering work by the ICARUS collaboration (see [5] and A. Guglielmi this conference) and the former LBNE collaboration. The active volume of $12 \mathrm{~m}$ high, $14.5 \mathrm{~m}$ wide and $58 \mathrm{~m}$ long, is instrumented with anode plane assemblies (APAs), which are $6 \mathrm{~m}$ high and $2.3 \mathrm{~m}$ wide, and cathode plane assemblies (CPAs), $3 \mathrm{~m}$ high by $2.3 \mathrm{~m}$ wide. Vertical stacks of two APAs and four CPAs instrument the $12 \mathrm{~m}$ height of the active volume. APAs and CPAs are separated horizontally by $3.6 \mathrm{~m}$. The CPAs are held at $180 \mathrm{kV}$, such that ionization electrons drift in an electric field of $500 \mathrm{~V} \cdot \mathrm{cm}^{-1}$, which leads to a maximum drift time of $2.3 \mathrm{~ms}$. A wire spacing of $5 \mathrm{~mm}$ requires 384,000 electronic readout channels. Planar photon detectors sensitive to the UV scintillation light, for event interaction time determination for underground physics, are mounted within the anode planes.

A dual-phase implementation of the DUNE far detector (which features extraction of track ionization from the liquid into the gas phase for amplification), developed by the former LBNO collaboration, has several potential benefits and is presented as an alternative design in the CDR. If the constructability, cost and performance are confirmed, it could form the basis of the second or subsequent 10-kt far detector modules.

A 35-tonne single-phase prototype has been constructed at Fermilab and a $1 \mathrm{~m} \times 1 \mathrm{~m} \times 3 \mathrm{~m}$ dual-phase prototype is under construction at the CERN. Two $8 \mathrm{~m} \times 8 \mathrm{~m} \times 8 \mathrm{~m}$ cryostats are planned at CERN to accommodate full-scale singleand double-phase LArTPCs and allow operation in a charged particle beam. 


\section{Summary and Conclusions}

In July 2015 LBNF/DUNE underwent a successful Conceptual Design review by the US Department of Energy, followed in December by a construction readiness review of the Far Site conventional facilities, which may lead to approval to start underground excavation at SURF in 2017. The goal is to begin installation of the first far detector module in 2021, leading to operation with the first $10 \mathrm{kt}$ for non-accelerator physics in 2023/4. It is anticipated that additional far detector modules will be commissioned about every 2 years. The neutrino beam, with a power of at least $1 \mathrm{MW}$, and the near detector would be operational by 2026 .

The International Long-Baseline Neutrino Facility hosted in the US along with the DUNE detectors will perform far-reaching measurements of CP violation, mass hierarchy, non-standard interactions, proton decay and supernova burst neutrinos from intra-galactic distances. DUNE is the only planned experiment able to provide $>5 \sigma$ determination of the mass ordering and for a favorable $\mathrm{CP}$ phase this could be achieved in a few years-intriguingly, current experiments seem to mildly favor this scenario. Progress to further internationalize the design, funding, construction and operation of the facility and the experimental program has proceeded very quickly. It is a challenging, longtimescale project but the science goals demand it!

\section{Acknowledgments}

The author would like to acknowledge the dedication and contributions of the members of the former LBNE Science Collaboration and Project staff who laid the groundwork for DUNE/LBNF. The work was supported by the US Department of Energy Office of High Energy Physics.

[1] S. Pascoli, S. Petcov, and A. Riotto, "Leptogenesis and Low Energy CP Violation in Neutrino Physics," Nucl.Phys. B774 (2007) 1-52.

[2] R. Acciarri et al., "Long-Baseline Neutrino Facility (LBNF) and Deep Underground Neutrino Experiment (DUNE) Conceptual Design Report Volume 2: The Physics Program for DUNE at LBNF," arXiv:1512.06148 [physics.ins-det], 2015 and see http://www.dunescience.org/.

[3] M. Bass et al., "Baseline optimization for the measurement of CP violation and mass hierarchy in a long-baseline neutrino oscillation experiment," Phys. Rev. D 91, 052015.

[4] DUNE Collaboration, "DUNE/LBNF CDR Volume 4: The DUNE Detectors at LBNF," arXiv:1601.02984 [physics.ins-det] 2016.

[5] S. Amerio et al., ICARUS Collaboration, "Design, construction and tests of the ICARUS T600 detector," Nucl.Inst.Meth. A527 (2004) 329-410. 